\title{
Review: CT angiography and magnetic resonance imaging are the best less invasive tests for renal artery stenosis
}

\author{
Vasbinder GB, Nelemans PJ, Kessels AG, et al. Diagnostic tests for renal artery stenosis in patients suspected of having \\ renovascular hypertension: a meta-analysis. Ann Intern Med 2001 Sep 18;135:401-11.
}

QUESTION: What are the diagnostic accuracies of 5 non-invasive or minimally invasive tests for detecting renal artery stenosis: computed tomographic angiography (CTA), magnetic resonance angiography (MRA), ultrasonography, captopril scintigraphy, and the captopril test?

\section{Data sources}

Studies were identified by searching Medline, EMBASE/ Excerpta Medica, and the Cochrane Library (to August 2000) and by scanning bibliographies of relevant studies.

\section{Study selection}

2 reviewers selected English, German, or French language studies if they used intra-arterial $\mathrm{x}$ ray angiography as the diagnostic standard, patients were referred for clinical suspicion of renovascular hypertension, criteria and cutoff points for positive results were explicitly defined for each test, and the number of positive and negative results were reported for both the test and the diagnostic standard. Exclusion criteria were patients with renal transplantation or possible verification bias (ie, patients with a positive result on the index test were referred to the diagnostic standard test more often than patients with a negative result).

\section{Data extraction}

Data were extracted on test, number of patients, definition of haemodynamically significant stenosis, use of blinding, whether accessory arteries were included, and sensitivity and specificity. Summary receiveroperating characteristic (ROC) curves were used to compare tests.

\section{Main results}

5 studies on CTA, 16 on MRA, 24 on ultrasonography, 14 on captopril renal scintigraphy, and 4 on the captopril test were included. The areas under the summary ROC curves were 0.99 for CTA, 0.99 for gadolinium-enhanced MRA, 0.97 for non-gadoliniumenhanced MRA, 0.93 for ultrasonography, 0.92 for captopril renal scintigraphy, and 0.72 for the captopril test. CTA and gadolinium-enhanced MRA were better than the other tests $(p<0.05)$ and were similar to each other. Non-gadolinium-enhanced MRA was better than captopril renal scintigraphy $(\mathrm{p}=0.022)$ and the captopril test $(\mathrm{p}<0.001)$. Ultrasonography $(\mathrm{p}=0.01)$ and captopril scintigraphy $(\mathrm{p}=0.017)$ were both better than the captopril test. Ultrasonography and captopril scintigraphy did not differ for diagnostic accuracy.

Dutch Health Car Insurance Board.

For correspondence: $\mathrm{Dr}$ $G$ B Vasbinder,

University Hospital of

Maastricht, Maastricht

The Netherlands.

bvas@rdia.amz.nl.

\section{Conclusion}

Computed tomographic angiography and gadoliniumenhanced magnetic resonance angiography have the best diagnostic accuracies for detecting renal stenosis. The captopril test has the worst diagnostic accuracy.

\section{COMMENTARY}

Internists often face the difficult decision of which patients with hypertension to investigate for renal artery stenosis. The meta-analysis by Vasbinder $e t a l$ is therefore a timely contribution to assess the diagnostic accuracy of noninvasive or minimally invasive screening tests for detecting renal artery stenosis. Their conclusion that CTA and gadolinium-enhanced MRA have the best diagnostic accuracy is certainly valid.

How generalisable are the results of the meta-analysis? First, many of the patients had atherosclerotic renal artery stenosis, often with accelerated hypertension, and renal insufficiency. Second, the choice of a specific screening test may be primarily determined by local availability, cost considerations, or concerns about side effects (eg, radiocontrast use in renal insufficiency). Most important, the association of renal artery stenosis with hypertension does not establish causation (ie, renovascular hypertension), and none of the screening tests examined in the meta-analysis nor renal angiography predicts a therapeutic response to revascularisation. The goal of treating renal artery stenosis is to cure or improve hypertension and to preserve renal function. Unfortunately, none of the revascularisation procedures have been proved to be more beneficial than medical treatment.

What should physicians considering a diagnosis of renovascular hypertension do? First, they can assess the likelihood of renal artery stenosis by using a simple clinical prediction rule. ${ }^{1}$ Patients with low likelihood $(<10 \%)$ should continue medical treatment. All other patients should be investigated only by physicians who have experience interpreting the clinical relevance of the results obtained with the diagnostic procedures described in the meta-analysis. Further evaluation should then focus on criteria predicting favourable outcomes ${ }^{2}$ and an in-depth discussion with the patient about the potential benefits and risks of revascularisation. Ultimately, randomised trials comparing renal stenting with medical treatment in preserving renal function are needed to guide clinicians in treating this complex disease.

Wolfgang J Weise, MD Jonathan B Jaffery, MD University of Vermont College of Medicine Burlington, Vermont, USA

1 Krijnen P, van Jaarsveld BC, Steyerberg EW, et al. A clinical prediction rule for renal artery stenosis. Ann Intern Med 1998;129:705-11.

2 Radermacher J, Chavan A, Bleck J, et al. Use of Doppler ultrasonography to predict the outcome of therapy for renal-artery stenosis. N Engl J Med 2001;344:410-7. 